Research Article

\title{
Hölder Continuity of a Parametric Generalized Variational Inequality
}

\author{
Li-na Wang ${ }^{1}$ and Xiao-bing $\mathrm{Li}^{2}$ \\ ${ }^{1}$ Chongqing Water Resources and Electric Engineering College, Chongqing 402160, China \\ ${ }^{2}$ College of Sciences, Chongqing Jiaotong University, Chongqing 400074, China \\ Correspondence should be addressed to Xiao-bing Li; xiaobinglicq@163.com
}

Received 17 January 2014; Accepted 10 March 2014; Published 22 April 2014

Academic Editor: Sheng-Jie Li

Copyright (c) 2014 L.-n. Wang and X.-b. Li. This is an open access article distributed under the Creative Commons Attribution License, which permits unrestricted use, distribution, and reproduction in any medium, provided the original work is properly cited.

By using the classic metric projection method, we obtain sufficient conditions for Hölder continuity of the nonunique solution mapping for a parametric generalized variational inequality with respect to data perturbation. The result is different from the recent ones in the literature and has a strong geometric flavor.

\section{Introduction}

Variational inequality is a very general mathematical format, which embraces the formats of several disciplines, as those for equilibrium problems of mathematical physics, those from game theory, and those for transportation equilibrium problems. Thus, it is important to derive results for parametric variational inequality concerning the properties of the solution mapping when the problem's data vary.

It is well known that the Hölder continuity of the perturbed solution mapping of variational inequalities is one aspect of stability analysis. In general, the stability analysis of solution mappings for parametric variational inequalities includes semicontinuity, Lipschitz continuity, and Hölder continuity of solution mappings. Most of the research in the area of stability analysis for variational inequalities has been performed under assumptions which implied the local uniqueness of perturbed solutions so that the solution mapping was single valued. By using the metric projection method, Dafermos [1] first derived sufficient conditions for the local uniqueness, continuity (or Lipschitz continuity), and differentiability of a perturbed solution of variational inequalities. Using the same techniques, Yen [2] and Yen and Lee [3] later obtained uniqueness of the solution for a classical perturbed variational inequality and showed that the solution mapping is Hölder continuous with respect to parameters.
Then, Domokos [4] extended these results of [1-3] to the case of reflexive Banach spaces.

There have also been a few papers to study more general situations where the solution sets of variational inequalities may be set valued. Robinson [5] investigated characterizations and existences of solutions for a generalized equation involving set-valued mappings under certain metric regularity hypotheses. As applications, he also studied some Lipschitz-type continuity property of the solution mapping for perturbed variational inequalities defined on closed convex sets. Ha [6] used the degree theory to derive some sufficient conditions, which guarantee the existence of nonunique perturbed solutions of nonlinear complementarity problems in a neighborhood of a reference point. Under the Hausdorff metric and the strong quasimonotonicity, Lee et al. [7] showed that nonunique solution mapping for a perturbed vector variational inequality is Hölder continuous with respect to parameters. Based on the scalarization technique and degree theoretic method, Wong [8] recently discussed the lower semicontinuity of the nonunique solution mapping for a perturbed vector variational inequality, where the operator may not be strongly monotone.

Although there have been many papers to study solution stability of perturbed variational inequalities, very few papers focus on such a study for perturbed generalized variational inequalities. Recently, by virtue of the strong quasimonotonicity, Ait Mansour and Aussel [9] have obtained a result 
on Hölder continuity of the nonunique solution mapping of a perturbed generalized variational inequality defined by strongly quasimonotone set-valued maps in the case of finite dimensional spaces. Without conditions related to the degree theory and the metric projection, Kien [10] derived sufficient conditions for the lower semicontinuity of nonunique perturbed solutions of a perturbed generalized variational inequality in reflexive Banach spaces.

Motivated by the work reported in $[1-4,9,10]$, our main interest is to investigate the Hölder continuity of nonunique perturbed solution mapping for a perturbed generalized variational inequality defined on perturbed feasible sets. We first introduce a locally strong monotone set-valued operator, which is weaker than the corresponding ones in $[1-4,9,10]$ and use the projection techniques of $[1,2,4]$ to derive some sufficient conditions, which guarantee the Hölder continuity of the locally nonunique solution sets for a perturbed generalized variational inequality with respect to parameters.

The rest of the paper is organized as follows. In Section 2, we introduce the parametric generalized variational inequality and recall the definitions and corresponding results which are needed in this paper. Then, we derive a relation between the Pseudo-Hölder property of a set-valued mapping and the Hölder property of projection mapping. In Section 3, we first introduce the key assumption $\left(\mathrm{H}_{2}\right)$ which is weaker than the corresponding ones in $[1-4,9,10]$ and the relative assumptions. Under these assumptions, we follow the projection technique of [1-4] mainly to study the behavior and Hölder property of the nonunique perturbed solution mapping for a parametric generalized variational inequality without the differentiability assumption and the degree theory. An example is also given to illustrate that our main result is applicable.

\section{Preliminaries}

Throughout this paper, if not other specified, let $E$ be a Hilbert space which is equipped with an inner product $\langle\cdot, \cdot\rangle$ and with the norm $\|\cdot\|$, respectively. Let $\Lambda, M$ be two parameter sets of the normed spaces, and let $B(x, \tau)$ denote the closed ball with the center $x$ and the radius $\tau$. The Hausdorff metric between two nonempty subsets $A, B$ of $E$ is defined by

$$
H(A, B):=\max \left\{\sup _{a \in A} d(a, B) \sup _{b \in B} d(A, b)\right\}
$$

where $d(a, B):=\inf _{b \in B}\|a-b\|$.

Let $K: \Lambda \rightrightarrows E$ be a set-valued mapping with nonempty closed convex values, and let $T: E \times M \rightrightarrows E$ be a setvalued mapping with nonempty compact values. Consider the following parametric generalized variational inequality consisting of finding $x \in K(\lambda)$ such that there exists $z^{*} \in$ $T(x, \mu)$ with

$$
\left\langle z^{*}, y-x\right\rangle \geq 0, \quad \forall y \in K(\lambda) .
$$

For each $(\lambda, \mu) \in \Lambda \times M$, the solution set of (2) is defined by

$$
\begin{aligned}
& S(\lambda, \mu):=\left\{x \in K(\lambda): \exists z^{*} \in T(x, \mu)\right. \\
&\text { with } \left.\left\langle z^{*}, y-x\right\rangle \geq 0, \forall y \in K(\lambda)\right\} .
\end{aligned}
$$

We first recall the following definitions and results which are needed in the sequel. Let $K$ be a nonempty closed convex subset of $E$, and let $P_{K}(z)$ denote the projection of $z \in E$ onto $K$. It is well known that the projection operator $P_{K}(z)$ is a nonexpansive operator. From [11], we have the following result.

Lemma 1. For each $z \in E, x=P_{K}(z)$ if and only if

$$
\langle x-z, y-x\rangle \geq 0, \quad \forall y \in K .
$$

Definition 2. Let $K: \Lambda \rightrightarrows E$ be a set-valued mapping with nonempty closed convex values, $\bar{\lambda} \in \Lambda$ and $\bar{x} \in K(\bar{\lambda}) . K(\cdot)$ is said to be $k$. $\alpha$-Pseudo-Hölder continuous at $(\bar{\lambda}, \bar{x})$ if and only if there exist a neighborhood $V$ of $\bar{\lambda}$, a neighborhood $W$ of $\bar{x}$ such that

$$
K\left(\lambda^{\prime}\right) \cap W \subset K(\lambda)+k\left\|\lambda^{\prime}-\lambda\right\|^{\alpha} B(0,1), \quad \forall \lambda, \lambda^{\prime} \in V \cap \Lambda,
$$

where $k>0, \alpha>0$.

Note that when $\alpha=1$, Definition 2 reduces to the Aubin property in [12].

From the definition of norm, we can easily obtain the following result.

Lemma 3. If $\alpha>0$, then the norm mapping $\|\cdot\|: E \rightarrow R$ satisfies

$$
\|x+y\|^{\alpha} \leq(\|x\|+\|y\|)^{\alpha} .
$$

Proof. If $x=0$ or $y=0$, the conclusion is trivial. Otherwise, $\|x+y\| /(\|x\|+\|y\|) \leq 1$ and $(\|x+y\| /(\|x\|+\|y\|))^{\alpha} \leq 1$. Hence, $\|x+y\|^{\alpha} \leq(\|x\|+\|y\|)^{\alpha}$.

The following Lemma, which is an extension of Lemma 1.1 in [2], plays an important role in this paper.

Lemma 4. Assume that $K(\cdot)$ is k. $\alpha$-Pseudo-Hölder continuous at $(\bar{\lambda}, \bar{x})$ and $X$ is a closed bounded convex neighborhood of $\bar{x}$. Then, there exist a neighborhood $X_{1}$ of $\bar{x}$, a neighborhood $V_{1}$ of $\bar{\lambda}$ such that $\forall y \in X_{1}, \forall \lambda, \lambda^{\prime} \in V_{1} \cap \Lambda$,

$$
\left\|P_{K(\lambda) \cap X}(y)-P_{K\left(\lambda^{\prime}\right) \cap X}(y)\right\| \leq 2 k\left\|\lambda-\lambda^{\prime}\right\|^{\alpha / 2} .
$$

Proof. We shall use the similar arguments of $[2,13]$ to prove the result.

Since $K(\cdot)$ is $k . \alpha$-Pseudo-Hölder continuous at $(\bar{\lambda}, \bar{x})$, there exist a neighborhood $W$ of $\bar{x}$, a neighborhood $V$ of $\bar{\lambda}$ which satisfy (5). Choose $\tau \in(0, k / 6)$ such that $B(\bar{x}, \tau) \subset$ $X \cap W$, and fix a number $\delta>0$ satisfying

$$
\delta \leq \min \left\{\frac{1}{2}\left(\frac{\tau}{4 k}\right)^{1 / \alpha},\left(\frac{\tau}{8 k}\right)^{1 / \alpha}\right\} .
$$


Let

$$
\begin{aligned}
& X_{1}:=B\left(\bar{x}, \frac{\tau}{8}\right) \cap B\left(\bar{x}, \frac{k}{8}\right), \\
& V_{1}:=V \cap B(\bar{\lambda}, \delta) .
\end{aligned}
$$

We claim that these $X_{1}, V_{1}$ satisfy (7). Indeed, since $B(\bar{x}, \tau) \subset X \cap W$ and $V_{1} \subset V$, then (5) implies that

$$
\begin{array}{r}
K\left(\lambda^{\prime}\right) \cap B(\bar{x}, \tau) \subset K(\lambda)+k\left\|\lambda-\lambda^{\prime}\right\|^{\alpha} B(0,1), \\
\forall \lambda, \lambda^{\prime} \in V_{1} \cap \Lambda .
\end{array}
$$

Furthermore, (5) implies

$$
\bar{x} \in K(\lambda)+k\|\lambda-\bar{\lambda}\|^{\alpha} B(0,1), \quad \forall \lambda \in V_{1} \cap \Lambda .
$$

Then, it follows from (8) and (10) that there exists $x \in K(\lambda)$ such that

$$
\|\bar{x}-x\| \leq k \delta^{\alpha} \leq k \frac{\tau}{8 k}=\frac{\tau}{8},
$$

which implies

$$
K(\lambda) \cap B\left(\bar{x}, \frac{\tau}{8}\right) \neq \emptyset, \quad \forall \lambda \in V_{1} \cap \Lambda .
$$

Let $x \in K(\lambda) \cap B(\bar{x}, \tau / 8)$. Then, it follows from (9) and the definition of projection operator that, for any $y \in X_{1}, \lambda \in$ $V_{1} \cap \Lambda$,

$$
\begin{aligned}
\left\|P_{K(\lambda) \cap X}(y)-\bar{x}\right\| & \leq\left\|P_{K(\lambda) \cap X}(y)-y\right\|+\|y-\bar{x}\| \\
& \leq\|x-y\|+\|y-\bar{x}\| \\
& \leq 2\|y-\bar{x}\|+\|x-\bar{x}\| \\
& \leq 2 \cdot \frac{\tau}{8}+\frac{\tau}{8}=\frac{3 \tau}{8}<\frac{\tau}{2},
\end{aligned}
$$

which means

$$
P_{K(\lambda) \cap X}(y) \in B\left(\bar{x}, \frac{\tau}{2}\right) .
$$

Assume to the contrary that (7) is false. Then, there exist $\lambda, \lambda^{\prime} \in V_{1} \cap \Lambda, y \in X_{1}$ such that

$$
\|a-b\|>2 k\left\|\lambda-\lambda^{\prime}\right\|^{\alpha / 2},
$$

where $a=P_{K(\lambda) \cap X}(y)$ and $b=P_{K\left(\lambda^{\prime}\right) \cap X}(y)$. From (16), we can easily see that

$$
a \in K(\lambda) \cap B\left(\bar{x}, \frac{\tau}{2}\right), \quad b \in K\left(\lambda^{\prime}\right) \cap B\left(\bar{x}, \frac{\tau}{2}\right) .
$$

Then, by virtue of (11), there exist $a_{1} \in K(\lambda)$ and $b_{1} \in K\left(\lambda^{\prime}\right)$ such that

$$
\left\|a-b_{1}\right\| \leq k\left\|\lambda-\lambda^{\prime}\right\|^{\alpha}, \quad\left\|b-a_{1}\right\| \leq k\left\|\lambda-\lambda^{\prime}\right\|^{\alpha} .
$$

Therefore, (8), (10), (11), (18), and (19) and Lemma 3 together yield that

$$
\begin{aligned}
\left\|a_{1}-\bar{x}\right\| & \leq\left\|a_{1}-b\right\|+\|b-\bar{x}\| \\
& \leq k\left\|\lambda-\lambda^{\prime}\right\|^{\alpha}+\|b-\bar{x}\| \\
& \leq k\left(\|\lambda-\bar{\lambda}\|+\left\|\bar{\lambda}-\lambda^{\prime}\right\|\right)^{\alpha}+\|b-\bar{x}\| \\
& \leq 2^{\alpha} k \delta^{\alpha}+\frac{\tau}{2} \leq \frac{3 \tau}{4}<\tau .
\end{aligned}
$$

This means that

$$
a_{1} \in K(\lambda) \cap B(\bar{x}, \tau) \subset K(\lambda) \cap X .
$$

Similarly, we have

$$
b_{1} \in K\left(\lambda^{\prime}\right) \cap B(\bar{x}, \tau) \subset K\left(\lambda^{\prime}\right) \cap X .
$$

Then, it follows from (19), (21), (22), and the definition of projection operator that

$$
\begin{aligned}
\|y-b\|-\|y-a\| & \leq\left\|y-b_{1}\right\|-\|y-a\| \\
& \leq\left\|a-b_{1}\right\| \leq k\left\|\lambda-\lambda^{\prime}\right\|^{\alpha}, \\
\|y-a\|-\|y-b\| & \leq\left\|y-a_{1}\right\|-\|y-b\| \\
& \leq\left\|b-a_{1}\right\| \leq k\left\|\lambda-\lambda^{\prime}\right\|^{\alpha} .
\end{aligned}
$$

Let $u:=\left\|y-a_{1}\right\|, v:=\|y-a\|$, and $w:=\left\|a_{1}-a\right\|$. From (19) and (23), we have

$$
\begin{aligned}
0 & \leq u-v \leq\left(\|y-b\|+\left\|b-a_{1}\right\|\right)-\|y-a\| \\
& \leq 2 k\left\|\lambda-\lambda^{\prime}\right\|^{\alpha} .
\end{aligned}
$$

Applying (17) and (19), we obtain

$$
\begin{aligned}
w & =\left\|a-a_{1}\right\| \geq\|a-b\|-\left\|b-a_{1}\right\| \\
& >2 k\left\|\lambda-\lambda^{\prime}\right\|^{\alpha / 2}-k\left\|\lambda-\lambda^{\prime}\right\|^{\alpha} .
\end{aligned}
$$

It follows from (8) and Lemma 3 that

$$
\left\|\lambda-\lambda^{\prime}\right\|^{\alpha} \leq\left(\|\lambda-\bar{\lambda}\|+\left\|\bar{\lambda}-\lambda^{\prime}\right\|\right)^{\alpha} \leq 2^{\alpha} \delta^{\alpha} \leq \frac{1}{24}<1 .
$$

Then,

$$
k\left\|\lambda-\lambda^{\prime}\right\|^{\alpha} \leq k\left\|\lambda-\lambda^{\prime}\right\|^{\alpha / 2},
$$

which together with (25) implies $w>k\left\|\lambda-\lambda^{\prime}\right\|^{\alpha / 2}$. Since $K(\lambda)$ is a convex subset of $E$ and $X$ is a convex neighborhood of $\bar{x}$, we have

$$
\left[a, a_{1}\right]:=\left\{\sigma a+(1-\sigma) a_{1}: \sigma \in[0,1]\right\} \subset K(\lambda) \cap X .
$$

Since $a=P_{K(\lambda) \cap X}(y)$, by the property of the projection, we have

$$
\langle y-a, a-x\rangle \geq 0, \quad \forall x \in K(\lambda) \cap X .
$$


Then, it follows that

$$
\begin{aligned}
u^{2} & =v^{2}+w^{2}+2\left\langle y-a, a-a_{1}\right\rangle \\
& >v^{2}+w^{2}>v^{2}+k^{2}\left\|\lambda-\lambda^{\prime}\right\|^{\alpha} .
\end{aligned}
$$

Hence, by (24),

$$
2 k\left\|\lambda-\lambda^{\prime}\right\|^{\alpha}(u+v) \geq u^{2}-v^{2}>k^{2}\left\|\lambda-\lambda^{\prime}\right\|^{\alpha}
$$

Since (17) implies that $\lambda \neq \lambda^{\prime}$, we have

$$
u+v>\frac{k}{2} \text {. }
$$

On the other hand, by virtue of (9), (18), and (21), we have

$$
\begin{aligned}
u+v & =\left\|y-a_{1}\right\|+\|y-a\| \\
& \leq 2\|y-\bar{x}\|+\left\|\bar{x}-a_{1}\right\|+\|\bar{x}-a\| \\
& \leq 2 \frac{k}{8}+\tau+\frac{\tau}{2}=\frac{k}{4}+\frac{3 \tau}{2} .
\end{aligned}
$$

As $\tau$ was chosen so that $\tau \in(0, k / 6)$, then

$$
u+v=\frac{k}{4}+\frac{3 \tau}{2}<\frac{k}{2}
$$

which is a contradiction to (32). The proof is complete.

Remark 5. Lemma 2.1 of [2] has (7) with $\alpha=1$. When $0<\alpha \leq$ 1 or $\alpha>1$; Lemma 4 is always satisfied. Therefore, Lemma 4 generalizes and improves Lemma 2.1 of [2].

\section{Main Results}

In this section, we always assume that $\bar{x} \in S(\bar{\lambda}, \bar{\mu})$ is a unique solution to (2) at given parameter $(\bar{\lambda}, \bar{\mu}) \in \Lambda \times M$. Let $X$ be a closed bounded convex neighborhood of $\bar{x}$, let $V$ be a neighborhood of $\bar{\lambda}$, and let $U$ be a neighborhood of $\bar{\mu}$. In order to analyze the behavior of the set-valued mapping $S(\lambda, \mu)$ around $S(\bar{\lambda}, \bar{\mu})$ when $(\lambda, \mu)$ is close to $(\bar{\lambda}, \bar{\mu})$, we need to consider the following restrict problem.

For each $(\lambda, \mu) \in(V \cap \Lambda) \times(U \cap M)$, find $x \in K(\lambda) \cap X$ such that there exists $z^{*} \in T(x, \mu)$ with

$$
\left\langle z^{*}, y-x\right\rangle \geq 0, \quad \forall y \in K(\lambda) \cap X
$$

Similarly, for $(\lambda, \mu) \in(V \cap \Lambda) \times(U \cap M)$, the local solution set of (2) is defined by

$$
\begin{aligned}
\operatorname{LS}(\lambda, \mu):= & \left\{x \in K(\lambda) \cap X: \exists z^{*} \in T(x, \mu)\right. \\
& \text { with } \left.\left\langle z^{*}, y-x\right\rangle \geq 0, \forall y \in K(\lambda) \cap X\right\} .
\end{aligned}
$$

To obtain our main result, we introduce the following assumptions for a neighborhood $V$ of $\bar{\lambda}$ and a neighborhood $U$ of $\bar{\mu}$.
$\left(\mathrm{H}_{1}\right)$ There exist $h>0, l>0$ and $\beta>0$ such that for any $t(\cdot, \cdot) \in T(\cdot, \cdot)$ satisfying

$$
\begin{array}{r}
\left\|t(x, \mu)-t\left(x^{\prime}, \mu^{\prime}\right)\right\| \leq h\left\|x-x^{\prime}\right\|+l\left\|\mu-\mu^{\prime}\right\|^{\beta}, \\
\forall x, x^{\prime} \in X \cap E, \quad \mu, \mu^{\prime} \in U \cap M,
\end{array}
$$

where $t: E \times M \rightarrow E$ is a vector-valued mapping.

$\left(\mathrm{H}_{2}\right)$ There exists $m>0$ such that for any $t(\cdot, \cdot) \in T(\cdot, \cdot)$ satisfying

$$
\begin{array}{r}
\left\langle t(x, \mu)-t\left(x^{\prime}, \mu\right), x-x^{\prime}\right\rangle \geq m\left\|x-x^{\prime}\right\|^{2}, \\
\forall x, x^{\prime} \in X \cap E, \quad \mu \in U \cap M,
\end{array}
$$

where $t: E \times M \rightarrow E$ is a vector-valued mapping.

$\left(\mathrm{H}_{3}\right)$ There exists $\rho>0$ such that

$$
\rho<\frac{m}{h^{2}} \text {. }
$$

Assumption $\left(\mathrm{H}_{1}\right)$ states $T(\cdot, \cdot)$ is locally strong h.1-l. $\beta$ Hölder continuous at $(\bar{x}, \bar{\mu})$, while assumption $\left(\mathrm{H}_{2}\right)$ is the requirement that $T(\cdot, \mu)$ is locally weak $m$-monotone at $\bar{x}$ with a coefficient independent to $\mu \in U \cap M$.

Remark 6. (1) If $T: E \times M \rightarrow E$ is a single-valued mapping and $\beta=1$, then assumptions $\left(\mathrm{H}_{1}\right)$ and $\left(\mathrm{H}_{2}\right)$ collapse to the locally Lipschitz at $(\bar{x}, \bar{\mu})$ and locally strongly monotone at $\bar{x}$ with a coefficient independent to $\mu \in U \cap M$ of [2], respectively.

(2) If $T(\cdot, \cdot)$ is a set-valued mapping with nonempty compact values, then for any $x, x^{\prime} \in X \cap E, \mu, \mu^{\prime} \in U \cap M$,

$$
\begin{aligned}
\left(\mathrm{H}_{1}\right) & \Longrightarrow H\left(T(x, \mu), T\left(x^{\prime}, \mu^{\prime}\right)\right) \\
& \leq h\left\|x-x^{\prime}\right\|+l\left\|\mu-\mu^{\prime}\right\|^{\beta} .
\end{aligned}
$$

(3) Obviously, assumption $\left(\mathrm{H}_{2}\right)$ is weaker than the following condition which was introduced in [14].

$\left(\mathrm{H}_{2}^{\prime}\right)$ For all $x, x^{\prime} \in X \cap E, \mu \in U \cap M$, there exists $m>0$ such that $\forall z^{*} \in T(x, \mu), z^{\prime *} \in T\left(x^{\prime}, \mu\right)$,

$$
\left\langle z^{*}-z^{\prime *}, x-x^{\prime}\right\rangle \geq m\left\|x-x^{\prime}\right\|^{2} .
$$

It is well know that $\left(\mathrm{H}_{2}^{\prime}\right)$ implies that the local solution mapping LS to (2) is single valued. However, when $\left(\mathrm{H}_{2}^{\prime}\right)$ is replaced by assumption $\left(\mathrm{H}_{2}\right)$, the local solution mapping $\mathrm{LS}$, in general, is not single valued; that is, LS may be a set-valued mapping.

(4) The following example is given to illustrate the existence of a class of set-valued mapping satisfying $\left(\mathrm{H}_{1}\right)$ and $\left(\mathrm{H}_{2}\right)$. It should be noted that the example also illustrates that $\left(\mathrm{H}_{2}\right)$ is weaker than $\left(\mathrm{H}_{2}^{\prime}\right)$ in [14].

Example 7. Let $E=\mathbb{R}$ and $M=[0,1]$. Let $T: E \times M \rightrightarrows E$ be a set-valued mapping which is defined by

$$
T(x, \mu)=\left\{t_{1}(x, \mu), t_{2}(x, \mu)\right\},
$$


where $t_{1}(x, \mu)=x-\mu$ and $t_{2}(x, \mu)=x+\mu$. Obviously, $T(x, \mu)$ is not single-valued mapping; $t_{1}(\cdot, \cdot)$ or $t_{2}(\cdot, \cdot)$ is 1.1-Lipschitz on $E \times M$; for any $\mu \in M, t_{1}(\cdot, \mu)$ or $t_{2}(\cdot, \mu)$ is 1-strongly monotone on $E$.

However, the set-valued mapping does not satisfy $\left(\mathrm{H}_{2}^{\prime}\right)$. Indeed, take $x, y \in E: x>y$ and $\mu \neq 0$. Then, we can easily see that $\left\langle t_{1}(x, \mu)-t_{2}(y, \mu), x-y\right\rangle=(x-y)^{2}-2 \mu(x-y)<$ $(x-y)^{2}$.

From Lemma 1 and the definition of the local solution for (2), we can get the following result.

Lemma 8. For each $(\lambda, \mu) \in(V \cap \Lambda) \times(U \cap M)$, the problem (2) has a local solution $x(\lambda, \mu)$ if and only if $x:=x(\lambda, \mu)$ is a fixed point of the set-valued mapping $G:(X \cap E) \times\left(V_{1} \cap \Lambda\right) \times$ $(U \cap M) \rightrightarrows X \cap E$ defined by

$$
G(x, \lambda, \mu):=\bigcup_{t(x, \mu) \in T(x, \mu)}\left\{P_{K(\lambda) \cap X}(x-\rho t(x, \mu))\right\},
$$

where $\rho>0$ is a fixed number.

Proof. Let $x(\lambda, \mu)$ be a local solution of (2) at parameters $\lambda \epsilon$ $V \cap \Lambda, \mu \in U \cap M$. Then, $x:=x(\lambda, \mu) \in K(\lambda) \cap X$ and $\bar{t}(x, \mu) \in T(x, \mu)$ exist such that

$$
\langle\bar{t}(x, \mu), y-x\rangle \geq 0, \quad \forall y \in K(\lambda) \cap X .
$$

Then, for any given $\rho>0$,

$$
\langle x-(x-\rho \bar{t}(x, \mu)), y-x\rangle \geq 0, \quad \forall y \in K(\lambda) \cap X,
$$

which along with Lemma 1 implies that

$$
\begin{aligned}
x & =P_{K(\lambda) \cap X}(x-\rho \bar{t}(x, \mu)) \\
& \in \bigcup_{t(x, \mu) \in T(x, \mu)}\left\{P_{K(\lambda) \cap X}(x-\rho t(x, \mu))\right\} .
\end{aligned}
$$

Therefore, $x(\lambda, \mu)$ is a fixed point of $G(\cdot, \lambda, \mu)$.

Since the converse can be similarly proved, we omit it.

Proposition 9. Suppose that assumptions $\left(H_{1}\right)-\left(H_{3}\right)$ hold. Then,

(a) for any $\lambda \in V \cap \Lambda, \mu \in U \cap M, G(\cdot, \lambda, \mu)$ defined by (43) is a set-valued $\theta$-contradiction mapping on $X \cap E$; that is, for any $x, x^{\prime} \in E \cap X$,

$$
H\left(G(x, \lambda, \mu), G\left(x^{\prime}, \lambda, \mu\right)\right) \leq \theta\|x-y\|
$$

where $\theta:=\sqrt{1-2 \rho m+\rho^{2} h^{2}}<1$;

(b) for any $\lambda \in V \cap \Lambda, \mu \in U \cap M$, the solution set to (2) is nonempty compact.

Proof. (a) For each $x \in X \cap E, \lambda \in V \cap \Lambda$, and $\mu \in U \cap M$, since $T(x, \mu)$ is a compact valued mapping and the projection mapping $P_{K(\lambda) \cap X}(\cdot)$ is continuous, then $G(x, \lambda, \mu)$ defined by (43) is a compact valued mapping. Therefore, the left part of
(47) is well defined. Let $a \in G(x, \lambda, \mu)$ be arbitrarily given. Then, there exists $\bar{t}(x, \mu) \in T(x, \mu)$ such that

$$
a=P_{K(\lambda) \cap X}(x-\rho \bar{t}(x, \mu)) .
$$

Let $b:=P_{K(\lambda) \cap X}\left(x^{\prime}-\rho \bar{t}\left(x^{\prime}, \mu\right)\right)$, which along with (43) yields that $b \in G\left(x^{\prime}, \lambda, \mu\right)$.

Using assumption $\left(\mathrm{H}_{1}\right)$ and the property that the projection onto a fixed closed convex subset is a nonexpansive mapping, we obtain

$$
\begin{aligned}
& \|a-b\| \\
& \quad=\left\|P_{K(\lambda) \cap X}(x-\rho \bar{t}(x, \mu))-P_{K(\lambda) \cap X}\left(x^{\prime}-\rho \bar{t}\left(x^{\prime}, \mu\right)\right)\right\| \\
& \quad \leq\left\|x-x^{\prime}-\rho\left(\bar{t}(x, \mu)-\bar{t}\left(x^{\prime}, \mu\right)\right)\right\| .
\end{aligned}
$$

On the other hand, it follows from assumptions $\left(\mathrm{H}_{1}\right)$ and $\left(\mathrm{H}_{2}\right)$ that

$$
\begin{aligned}
\| x-x^{\prime} & -\rho\left(\bar{t}(x, \mu)-\bar{t}\left(x^{\prime}, \mu\right)\right) \|^{2} \\
= & \left\|x-x^{\prime}\right\|^{2}-2 \rho\left\langle\bar{t}(x, \mu)-\bar{t}\left(x^{\prime}, \mu\right), x-x^{\prime}\right\rangle \\
& +\rho^{2}\left\|\bar{t}(x, \mu)-\bar{t}\left(x^{\prime}, \mu\right)\right\|^{2} \\
\leq & \left(1-2 \rho m+\rho^{2} h^{2}\right)\left\|x-x^{\prime}\right\|^{2},
\end{aligned}
$$

which implies that

$$
\begin{gathered}
\left\|x-x^{\prime}-\rho\left(\bar{t}(x, \mu)-\bar{t}\left(x^{\prime}, \mu\right)\right)\right\| \\
\leq \sqrt{1-2 \rho m+\rho^{2} h^{2}}\left\|x-x^{\prime}\right\| .
\end{gathered}
$$

Combing (49) and (51), we obtain

$$
\|a-b\| \leq \sqrt{1-2 \rho m+\rho^{2} h^{2}}\left\|x-x^{\prime}\right\|=\theta\left\|x-x^{\prime}\right\| .
$$

By assumption $\left(\mathrm{H}_{3}\right), \theta<1$ and hence we have

$$
\sup _{a \in G(x, \lambda, \mu)} d\left(a, G\left(x^{\prime}, \lambda, \mu\right)\right) \leq \theta\left\|x-x^{\prime}\right\| .
$$

Using the same arguments, we can show that

$$
\sup _{b \in G\left(x^{\prime}, \lambda, \mu\right)} d(b, G(x, \lambda, \mu)) \leq \theta\left\|x-x^{\prime}\right\|,
$$

which along with (53) implies that $G(\cdot, \lambda, \mu)$ is a set-valued $\theta$-contradiction mapping on $X \cap E$.

(b) By (a) and the Nadler fixed point theorem in [15], $G(\cdot, \lambda, \mu)$ has a fixed point $x(\lambda, \mu)$ for each $(\lambda, \mu) \in(V \cap$ $\Lambda) \times(U \cap M)$. Hence, for any $(\lambda, \mu) \in(V \cap \Lambda) \times(U \cap M)$, $\operatorname{LS}(\lambda, \mu) \neq \emptyset$ by Lemma 8 . Moreover, we claim that $\operatorname{LS}(\lambda, \mu)$ is a closed subset. Indeed, let $x_{n} \in \operatorname{LS}(\lambda, \mu)$ with $x_{n} \rightarrow x_{0}$ as $n \rightarrow \infty$. Then, it follows from Lemma 8 that

$$
x_{n} \in G\left(x_{n}, \lambda, \mu\right), \quad \forall n .
$$


Therefore, by (a), we obtain

$$
\begin{aligned}
& d\left(x_{0}, G\left(x_{0}, \lambda, \mu\right)\right) \\
& \leq\left\|x_{0}-x_{n}\right\|+d\left(x_{n}, G\left(x_{n}, \lambda, \mu\right)\right) \\
& \quad+H\left(G\left(x_{n}, \lambda, \mu\right), G\left(x_{0}, \lambda, \mu\right)\right) \\
& \leq(1+\theta)\left\|x_{0}-x_{n}\right\| \longrightarrow 0, \quad \text { as } n \longrightarrow \infty,
\end{aligned}
$$

which implies that $x_{0} \in G\left(x_{0}, \lambda, \mu\right)$ and $x_{0} \in \operatorname{LS}(\lambda, \mu)$ by Lemma 8. Hence, $\operatorname{LS}(\lambda, \mu)$ is a compact set since $K(\lambda) \cap X$ is compact. The proof is complete.

As an immediate consequence of Lemma 8, Proposition 9, and the Banach fixed point theorem, we have the following result.

Corollary 10. For any $(\lambda, \mu) \in(V \cap \Lambda) \times(U \cap M), t(\cdot, \mu) \in$ $T(\cdot, \mu)$, the vector-valued mapping $g:(X \cap E) \times\left(V_{1} \cap \Lambda\right) \times$ $(U \cap M) \rightarrow X \cap$ E defined by

$$
g(x, \lambda, \mu):=P_{K(\lambda) \cap X}(x-\rho t(x, \mu)),
$$

where $\rho>0$, is a fixed number. Furthermore, assume that assumptions $\left(\mathrm{H}_{1}\right)-\left(\mathrm{H}_{3}\right)$ are satisfied.

Then, for any $\lambda \in V \cap \Lambda, \mu \in U \cap M, g(\cdot, \lambda, \mu)$ is a vectorvalued $\theta$-contradiction mapping and has a unique fixed point $x(\lambda, \mu)$.

Now, we state our main result.

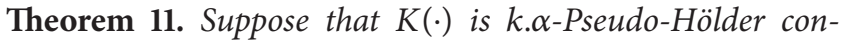
tinuous at $(\bar{\lambda}, \bar{x})$. Furthermore, suppose that assumptions $\left(H_{1}\right)-\left(H_{3}\right)$ hold. Then, there exist a neighborhood $\bar{U}$ of $\bar{\mu}$, a neighborhood $\bar{V}$ of $\bar{\lambda}$ such that

(a) for all $\left(\lambda^{\prime}, \mu^{\prime}\right),(\lambda, \mu) \in(\bar{V} \cap \Lambda) \times(\bar{U} \cap M)$,

$$
\begin{aligned}
& H\left(L S\left(\lambda^{\prime}, \mu^{\prime}\right), L S(\lambda, \mu)\right) \\
& \quad \leq \frac{1}{1-\theta}\left(2 k\left\|\lambda-\lambda^{\prime}\right\|^{\alpha / 2}+\rho l\left\|\mu-\mu^{\prime}\right\|^{\beta}\right) ;
\end{aligned}
$$

(b) for all $(\lambda, \mu) \in(\bar{V} \cap \Lambda) \times(\bar{U} \cap M), L S(\lambda, \mu) \subset$ int $X$ and for any $x(\lambda, \mu) \in L S(\lambda, \mu)$ is a solution to (2) at parameters $\lambda \in \bar{V} \cap \Lambda, \mu \in \bar{U} \cap M$.

Proof. By the Pseudo-Hölder continuity of $K(\cdot)$ and Lemma 4 , there exist a neighborhood $V_{1}$ of $\bar{\lambda}$, a neighborhood $X_{1}$ of $\bar{x}$ such that

$$
\begin{aligned}
& \left\|P_{K\left(\lambda^{\prime}\right) \cap X}(y)-P_{K(\lambda) \cap X}(y)\right\| \\
& \quad \leq 2 k\left\|\lambda^{\prime}-\lambda\right\|^{\alpha / 2}, \quad \forall \lambda, \lambda^{\prime} \in V_{1} \cap \Lambda, \forall y \in X_{1} .
\end{aligned}
$$

Since $T(\bar{x}, \bar{\mu})$ is compact, then $\sigma$ := $\sup _{t(\bar{x}, \bar{\mu}) \in T(\bar{x}, \bar{\mu})}\|t(\bar{x}, \bar{\mu})\|<\infty$. Let $\rho_{1}$ satisfy assumption $\left(\mathrm{H}_{3}\right)$ and

$$
\bar{x}+\rho_{1} \sigma B(0,1) \subset \operatorname{int} X_{1},
$$

where int $X_{1}$ denotes the interior of $X_{1}$.
Fix $\rho \in\left(0, \rho_{1}\right]$. For any $(\lambda, \mu) \in\left(V_{1} \cap \Lambda\right) \times(U \cap M)$, let $x \in \operatorname{LS}(\lambda, \mu)$ be arbitrarily given. Then, there exists $\widehat{t}(x, \mu) \in$ $T(x, \mu)$ such that

$$
x=P_{K(\lambda) \cap X}(x-\rho \hat{t}(x, \mu)) .
$$

For any $\left(\lambda^{\prime}, \mu^{\prime}\right) \in\left(V_{1} \cap \Lambda\right) \times(U \cap M)$, by Corollary 10, $g\left(\cdot, \lambda^{\prime}, \mu^{\prime}\right)=P_{K\left(\lambda^{\prime}\right) \cap X}(\cdot-\rho \widehat{t}(\cdot, \mu))$ has a unique fixed point $x^{\prime}=$ $x\left(\lambda^{\prime}, \mu^{\prime}\right)$. Then, it follows from Lemma 8 that $x^{\prime}=x\left(\lambda^{\prime}, \mu^{\prime}\right)$ is a solution to (2) at parameters $\left(\lambda^{\prime}, \mu^{\prime}\right)$; that is,

$$
x^{\prime}=P_{K\left(\lambda^{\prime}\right) \cap X}\left(x^{\prime}-\rho \widehat{t}\left(x^{\prime}, \mu^{\prime}\right)\right) .
$$

Then, using assumption $\left(\mathrm{H}_{1}\right)$, Proposition 9, and the property that the projection onto a fixed convex subset is a nonexpansive mapping, we obtain

$$
\begin{aligned}
\left\|x-x^{\prime}\right\|= & \left\|P_{K(\lambda) \cap X}(z(x, \mu))-P_{K\left(\lambda^{\prime}\right) \cap X}\left(z\left(x^{\prime}, \mu^{\prime}\right)\right)\right\| \\
\leq & \left\|P_{K(\lambda) \cap X}(z(x, \mu))-P_{K(\lambda) \cap X}\left(z\left(x, \mu^{\prime}\right)\right)\right\| \\
& +\left\|P_{K(\lambda) \cap X}\left(z\left(x, \mu^{\prime}\right)\right)-P_{K(\lambda) \cap X}\left(z\left(x^{\prime}, \mu^{\prime}\right)\right)\right\| \\
& +\left\|P_{K(\lambda) \cap X}\left(z\left(x^{\prime}, \mu^{\prime}\right)\right)-P_{K\left(\lambda^{\prime}\right) \cap X}\left(z\left(x^{\prime}, \mu^{\prime}\right)\right)\right\| \\
\leq & \left\|z(x, \mu)-z\left(x, \mu^{\prime}\right)\right\|+\theta\|x-\bar{x}\| \\
& +\left\|P_{K(\lambda) \cap X}\left(z\left(x^{\prime}, \mu^{\prime}\right)\right)-P_{K\left(\lambda^{\prime}\right) \cap X}\left(z\left(x^{\prime}, \mu^{\prime}\right)\right)\right\| \\
\leq & \rho\left\|\hat{t}(x, \mu)-\widehat{t}\left(x, \mu^{\prime}\right)\right\|+\theta\left\|x-x^{\prime}\right\| \\
& +\left\|P_{K(\lambda) \cap X}\left(z\left(x^{\prime}, \mu^{\prime}\right)\right)-P_{K\left(\lambda^{\prime}\right) \cap X}\left(z\left(x^{\prime}, \mu^{\prime}\right)\right)\right\| \\
\leq & \rho l\left\|\mu-\mu^{\prime}\right\|^{\beta}+\theta\left\|x-x^{\prime}\right\| \\
& +\left\|P_{K(\lambda) \cap X}\left(z\left(x^{\prime}, \mu^{\prime}\right)\right)-P_{K\left(\lambda^{\prime}\right) \cap X}\left(z\left(x^{\prime}, \mu^{\prime}\right)\right)\right\|,
\end{aligned}
$$

where $z(x, \mu):=x-\rho \widehat{t}(x, \mu), z\left(x^{\prime}, \mu^{\prime}\right):=x^{\prime}-\rho \widehat{t}\left(x^{\prime}, \mu^{\prime}\right)$, $z\left(x, \mu^{\prime}\right):=x-\rho \widehat{t}\left(x, \mu^{\prime}\right)$, and $\theta<1$ defined as in Proposition 9.

Noting that the arbitrariness of $x \in \operatorname{LS}(\lambda, \mu)$, we obtain that

$$
\begin{aligned}
& \sup _{x \in \operatorname{LS}(\lambda, \mu)} d\left(x, \operatorname{LS}\left(\lambda^{\prime}, \mu^{\prime}\right)\right) \\
& \leq \frac{1}{1-\theta}\left(\rho l\left\|\mu-\mu^{\prime}\right\|^{\beta}+\| P_{K(\lambda) \cap X}\left(z\left(x^{\prime}, \mu^{\prime}\right)\right)\right. \\
&\left.\quad-P_{K\left(\lambda^{\prime}\right) \cap X}\left(z\left(x^{\prime}, \mu^{\prime}\right)\right) \|\right) .
\end{aligned}
$$


Then, substituting $\left(\lambda^{\prime}, \mu^{\prime}\right)=(\bar{\lambda}, \bar{\mu})$ in $(64)$ and by the uniqueness of $\operatorname{LS}(\bar{\lambda}, \bar{\mu})$ we obtain that

$$
\begin{aligned}
& \sup _{x \in \operatorname{LS}(\lambda, \mu)} d(x, \operatorname{LS}(\bar{\lambda}, \bar{\mu}))= \sup _{x \in \operatorname{LS}(\lambda, \mu)}\|x-\bar{x}\| \\
& \leq \frac{1}{1-\theta}\left(\rho l\|\mu-\bar{\mu}\|^{\beta}\right. \\
&+\| P_{K(\lambda) \cap X}(z(\bar{x}, \bar{\mu})) \\
&\left.\quad-P_{K(\bar{\lambda}) \cap X}(z(\bar{x}, \bar{\mu})) \|\right),
\end{aligned}
$$

where $z(\bar{x}, \bar{\mu}):=\bar{x}-\rho \widehat{t}(\bar{x}, \bar{\mu})$.

According to (60), we have $z(\bar{x}, \bar{\mu}):=\bar{x}-\rho \widehat{t}(\bar{x}, \bar{\mu}) \in$ int $X_{1}$, which along with (59), (65) yields that

$$
\begin{aligned}
H(\operatorname{LS}(\lambda, \mu),\{\bar{x}\}) & =H(\operatorname{LS}(\lambda, \mu), \operatorname{LS}(\bar{\lambda}, \bar{\mu})) \\
& \leq \frac{1}{1-\theta}\left(2 k\|\lambda-\bar{\lambda}\|^{\alpha / 2}+\rho l\|\mu-\bar{\mu}\|^{\beta}\right) .
\end{aligned}
$$

We claim that there exist a neighborhood $\bar{V}$ of $\bar{\lambda}$, a neighborhood $\bar{U}$ of $\bar{\lambda}$ such that (58) is satisfied. Indeed, let $\epsilon>0$ be so small that $z(\bar{x}, \bar{\mu})+\epsilon B(0,1) \subset \operatorname{int} X_{1}$. Using (66), assumption $\left(\mathrm{H}_{1}\right)$ and (5) of Remark 6, we can find a neighborhood $\bar{V} \subset V_{1}$ of $\bar{\lambda}$, a neighborhood $\bar{U} \subset U$ of $\bar{\mu}$ such that for any $(\lambda, \mu) \in(\bar{V} \cap \Lambda) \times(\bar{U} \cap M)$,

$$
\begin{array}{r}
\operatorname{LS}(\lambda, \mu) \subset \operatorname{int} X, \quad H(x-\rho T(x, \mu), \bar{x}-\rho T(\bar{x}, \bar{\mu})) \leq \epsilon, \\
\forall x \in \operatorname{LS}(\lambda, \mu) .
\end{array}
$$

Therefore, (60) and (67) together yield that, for all $x \in$ $\operatorname{LS}(\lambda, \mu), t(x, \mu) \in T(x, \mu)$,

$$
z(\lambda, \mu):=x-\rho t(x, \mu) \in \operatorname{int} X_{1} .
$$

Hence, for any $(\lambda, \mu),\left(\lambda^{\prime}, \mu^{\prime}\right) \in(\bar{V} \cap \Lambda) \times(\bar{U} \cap M)$, it follows from (59), (64), and (68) that

$$
\begin{aligned}
& \sup _{x \in \operatorname{LS}(\lambda, \mu)} d\left(x, \operatorname{LS}\left(\lambda^{\prime}, \mu^{\prime}\right)\right) \\
& \quad \leq \frac{1}{1-\theta}\left(2 k\left\|\lambda-\lambda^{\prime}\right\|^{\alpha / 2}+\rho l\left\|\mu-\mu^{\prime}\right\|^{\beta}\right) .
\end{aligned}
$$

On the other hand, it follows from the symmetrical roles of $(\lambda, \mu),\left(\lambda^{\prime}, \mu^{\prime}\right)$ that

$$
\begin{aligned}
& \sup _{x \in \operatorname{LS}\left(\lambda^{\prime}, \mu^{\prime}\right)} d(x, \operatorname{LS}(\lambda, \mu)) \\
& \quad \leq \frac{1}{1-\theta}\left(2 k\left\|\lambda-\lambda^{\prime}\right\|^{\alpha / 2}+\rho l\left\|\mu-\mu^{\prime}\right\|^{\beta}\right) .
\end{aligned}
$$

Therefore, (69) and (70) together yield that for any $(\lambda, \mu)$, $\left(\lambda^{\prime}, \mu^{\prime}\right) \in(\bar{V} \cap \Lambda) \times(\bar{U} \cap M)$,

$$
\begin{aligned}
& H\left(\operatorname{LS}(\lambda, \mu), \operatorname{LS}\left(\lambda^{\prime}, \mu^{\prime}\right)\right) \\
& \quad \leq \frac{1}{1-\theta}\left(2 k\left\|\lambda-\lambda^{\prime}\right\|^{\alpha / 2}+\rho l\left\|\mu-\mu^{\prime}\right\|^{\beta}\right) .
\end{aligned}
$$

Thus, we have established (58) and obtained $\operatorname{LS}(\lambda, \mu) \subset$ int $X$ in (67), which implies that for all $x(\lambda, \mu) \in \operatorname{LS}(\lambda, \mu)$ is the solution in $X$ to (2) at parameters $\lambda \in \bar{V} \cap \Lambda, \mu \in \bar{U} \cap M$. The proof is complete.

The following example is given to illustrate that the local solution set of (2) is not single valued and Theorem 11 is applicable.

Example 12. Let $E=\mathbb{R}^{2}, \Lambda=M=\mathbb{R},(\bar{\lambda}, \bar{\mu})=(1,0) \in \Lambda \times M$ be a given point and let $V=\Lambda, U=M$ be a neighborhood of $(\bar{\lambda}, \bar{\mu})$. Let $T: E \times M \rightrightarrows E$ be a set-valued mapping which is defined by

$$
\begin{array}{r}
T(x, \mu)=\left\{\left((2-\theta) x_{1},(2-\theta) x_{2}+(1-2 \theta) \mu\right): 0 \leq \theta \leq 1\right\} \\
\forall x=\left(x_{1}, x_{2}\right) \in E, \quad \mu \in M
\end{array}
$$

and $K: \Lambda \rightrightarrows E$ defined by

$$
K(\lambda)=\left\{x=\left(x_{1}, x_{2}\right) \in E: x_{1}+x_{2}=2 \lambda\right\}, \quad \forall \lambda \in \Lambda
$$

Clearly, $\bar{x}=(1,1)$ is a unique solution to (2) at parameter $(\bar{\lambda}, \bar{\mu})$. Let $X=\mathbb{R}^{2}$ be a neighborhood of $\bar{x}$ and let $t(\cdot, \cdot) \in$ $T(\cdot, \cdot)$ be taken arbitrarily. Obviously, $t(\cdot, \cdot)$ is 2.1-1.1-Hölder continuous and strongly 1-monotone on $(X \cap E) \times(U \cap M)$; there exists $\rho$ satisfying $0<\rho \leq 1 / 4$; the map $K(\cdot)$ is 1.1-Hölder continuous with closed convex values on $V \cap \Lambda$. Therefore, all assumptions of Theorem 11 are satisfied.

Moreover, by virtue of Lemma 8 , we note that $x \in K(\lambda) \cap$ $X$ is a local solution of (2) at parameter $(\lambda, \mu)$ if and only if $x$ satisfies the inclusion

$$
x \in G(x, \lambda, \mu)=\bigcup_{t(x, \mu) \in T(x, \mu)}\left\{P_{K(\lambda) \cap X}(x-\rho t(x, \mu))\right\} .
$$

Let $x=\left(x_{1}, x_{2}\right) \in E$ be any point. By a simple computation, we obtain

$$
P_{K(\lambda) \cap X}(x)=\left(\lambda+\frac{x_{1}-x_{2}}{2}, \lambda+\frac{x_{2}-x_{1}}{2}\right) .
$$

Then, it follows that, for each $\rho: 0<\rho \leq 1 / 4$ and any $t(x, \mu) \in$ $T(x, \mu)$,

$$
\begin{gathered}
P_{K(\lambda) \cap X}(x-\rho t(x, \mu)) \\
=\left(\lambda+\left(\left((1-\rho(2-\theta)) x_{1}-(1-\rho(2-\theta)) x_{2}\right.\right.\right. \\
\left.-\rho(1-2 \theta) \mu)) \times 2^{-1}\right), \\
\lambda+\left(\left((1-\rho(2-\theta)) x_{2}+\rho(1-2 \theta) \mu\right.\right. \\
\left.\left.\left.-(1-\rho(2-\theta)) x_{1}\right) \times 2^{-1}\right)\right) .
\end{gathered}
$$


Hence,

$$
\begin{aligned}
& x \in G(x, \lambda, \mu) \\
&= \bigcup_{t(x, \mu) \in T(x, \mu)} P_{K(\lambda) \cap X}(x-\rho t(x, \mu)) \\
&=\left\{\left(\lambda+\left(\left((1-\rho(2-\theta)) x_{1}-(1-\rho(2-\theta)) x_{2}\right.\right.\right.\right. \\
&\left.\quad-\rho(1-2 \theta) \mu)) \times 2^{-1}\right), \\
& \quad \lambda+\left(\left((1-\rho(2-\theta)) x_{2}+\rho(1-2 \theta) \mu\right.\right. \\
&\left.\left.\left.\quad-(1-\rho(2-\theta)) x_{1}\right) \times 2^{-1}\right): 0 \leq \theta \leq 1\right\} .
\end{aligned}
$$

By virtue of Lemma 8, direct computation shows that, for any $(\lambda, \mu) \in(V \cap \Lambda) \times(U \cap M)$,

$$
\operatorname{LS}(\lambda, \mu)=\left\{\left(\lambda+\frac{(1-2 \theta) \mu}{2(2-\theta)}, \lambda-\frac{(1-2 \theta) \mu}{2(2-\theta)}\right): 0 \leq \theta \leq 1\right\} .
$$

Then, the local solution set $\operatorname{LS}(\cdot, \cdot)$ of (2) satisfies (58) for any neighborhoods $V$ of $\bar{\lambda}, U$ of $\bar{\mu}$. Hence, Theorem 11 is applicable.

\section{Conflict of Interests}

The authors declare that they have no conflict of interests regarding the publication of this paper.

\section{Acknowledgments}

The authors would like to express their deep gratitude to the anonymous referees and the associate editor for their valuable comments and suggestions which helped to improve the paper. This research was partially supported by the National Natural Science Foundation of China (Grant no. 11201509).

\section{References}

[1] S. Dafermos, "Sensitivity analysis in variational inequalities," Mathematics of Operations Research, vol. 13, no. 3, pp. 421-434, 1988.

[2] N. D. Yen, "Hölder continuity of solutions to a parametric variational inequality," Applied Mathematics and Optimization, vol. 31, no. 3, pp. 245-255, 1995.

[3] N. D. Yen and G. M. Lee, "Solution sensitivity of a class of variational inequalities," Journal of Mathematical Analysis and Applications, vol. 215, no. 1, pp. 48-55, 1997.

[4] A. Domokos, "Solution sensitivity of variational inequalities," Journal of Mathematical Analysis and Applications, vol. 230, no. 2, pp. 382-389, 1999.

[5] S. M. Robinson, "Generalized equations and their solutions. I: basic theory," Mathematical Programming Study, no. 10, pp. 128$141,1979$.

[6] C. D. Ha, "Application of degree theory in stability of the complementarity problem," Mathematics of Operations Research, vol. 12, no. 2, pp. 368-376, 1987.
[7] G. M. Lee, D. S. Kim, B. S. Lee, and N. D. Yen, "Vector variational inequality as a tool for studying vector optimization problems," Nonlinear Analysis: Theory, Methods \& Applications, vol. 34, no. 5, pp. 745-765, 1998.

[8] M. M. Wong, "Lower semicontinuity of the solution map to a parametric vector variational inequality," Journal of Global Optimization, vol. 46, no. 3, pp. 435-446, 2010.

[9] M. Ait Mansour and D. Aussel, "Quasimonotone variational inequalities and quasiconvex programming: quantitative stability," Pacific Journal of Optimization, vol. 2, no. 3, pp. 611-626, 2006.

[10] B. T. Kien, "Lower semicontinuity of the solution map to a parametric generalized variational inequality in reflexive Banach spaces," Set-Valued Analysis, vol. 16, no. 7-8, pp. 10891105, 2008.

[11] D. Kinderlehrer and G. Stampacchia, An Introduction to Variational Inequalities and Their Applications, vol. 88, Academic Press, New York, NY, USA, 1980.

[12] J.-P. Aubin and I. Ekeland, Applied Nonlinear Analysis, John Wiley \& Sons, New York, NY, USA, 1984.

[13] H. Attouch and R. J.-B. Wets, "Quantitative stability of variational systems. II. A framework for nonlinear conditioning," SIAM Journal on Optimization, vol. 3, no. 2, pp. 359-381, 1993.

[14] X. P. Ding and C. L. Luo, "On parametric generalized quasivariational inequalities," Journal of Optimization Theory and Applications, vol. 100, no. 1, pp. 195-205, 1999.

[15] S. B. Nadler, "Multivalued contraction mappings," Pacific Journal of Mathematics, vol. 30, pp. 475-485, 1969. 


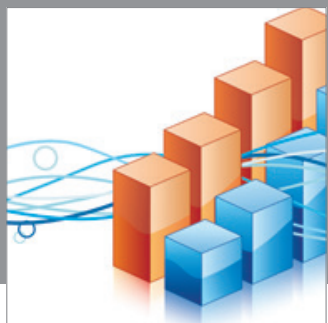

Advances in

Operations Research

mansans

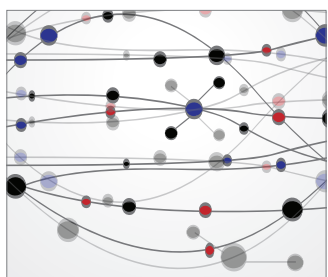

The Scientific World Journal
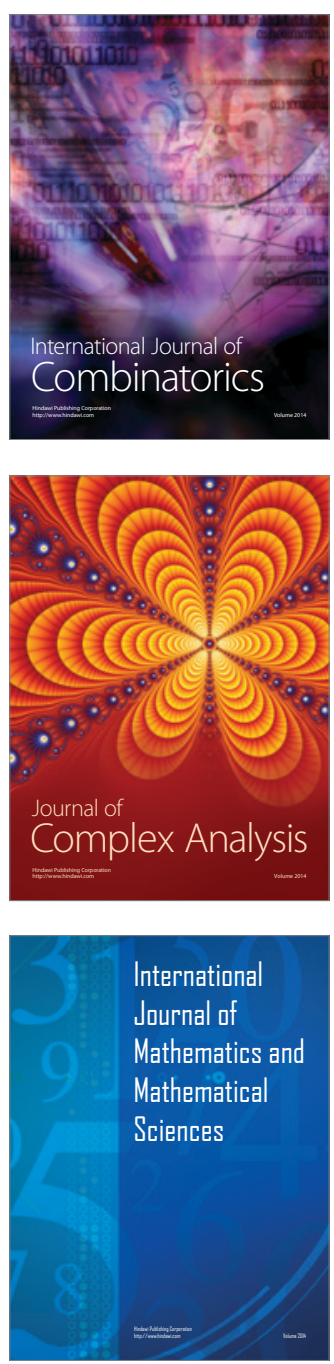
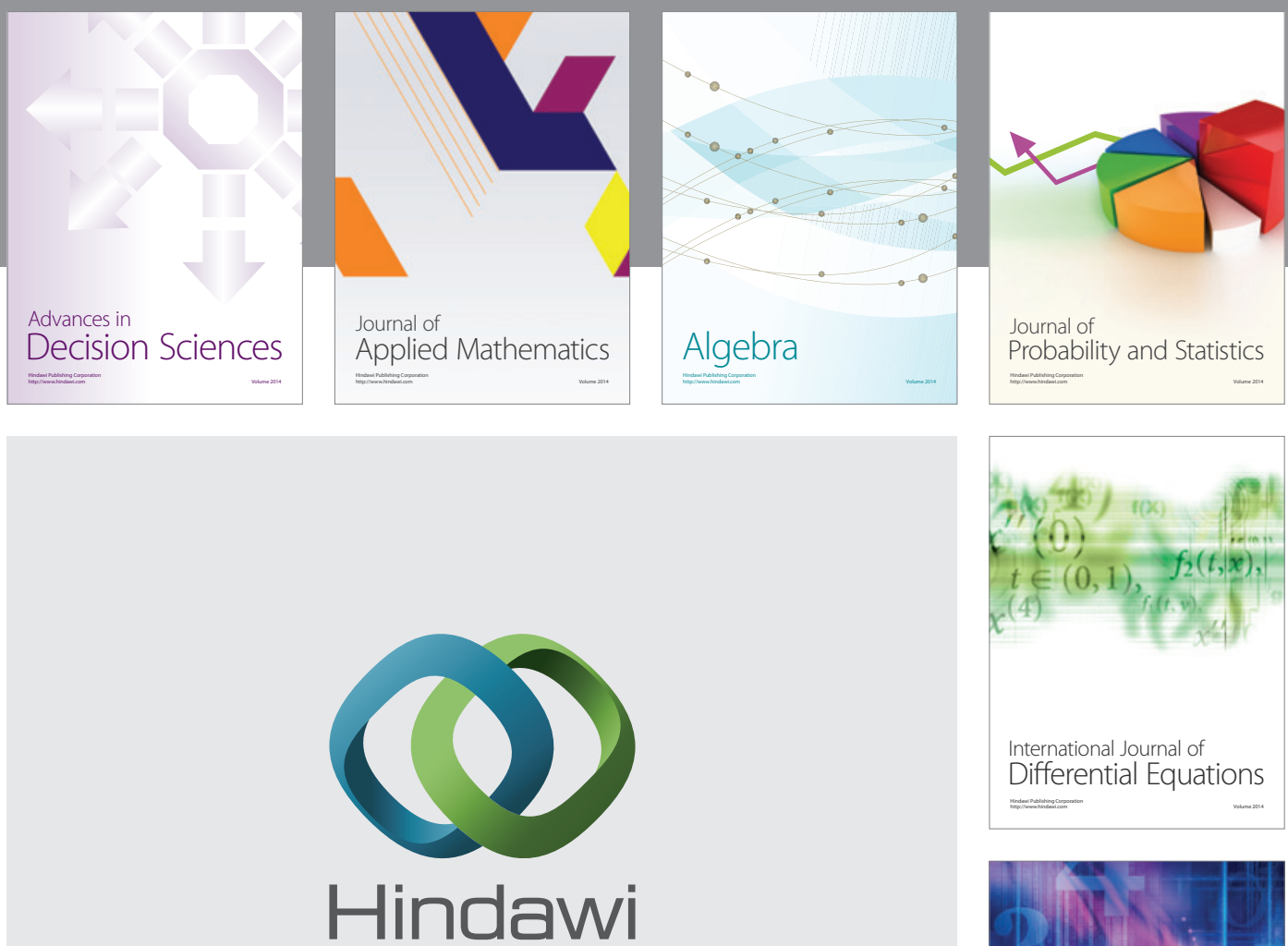

Submit your manuscripts at http://www.hindawi.com
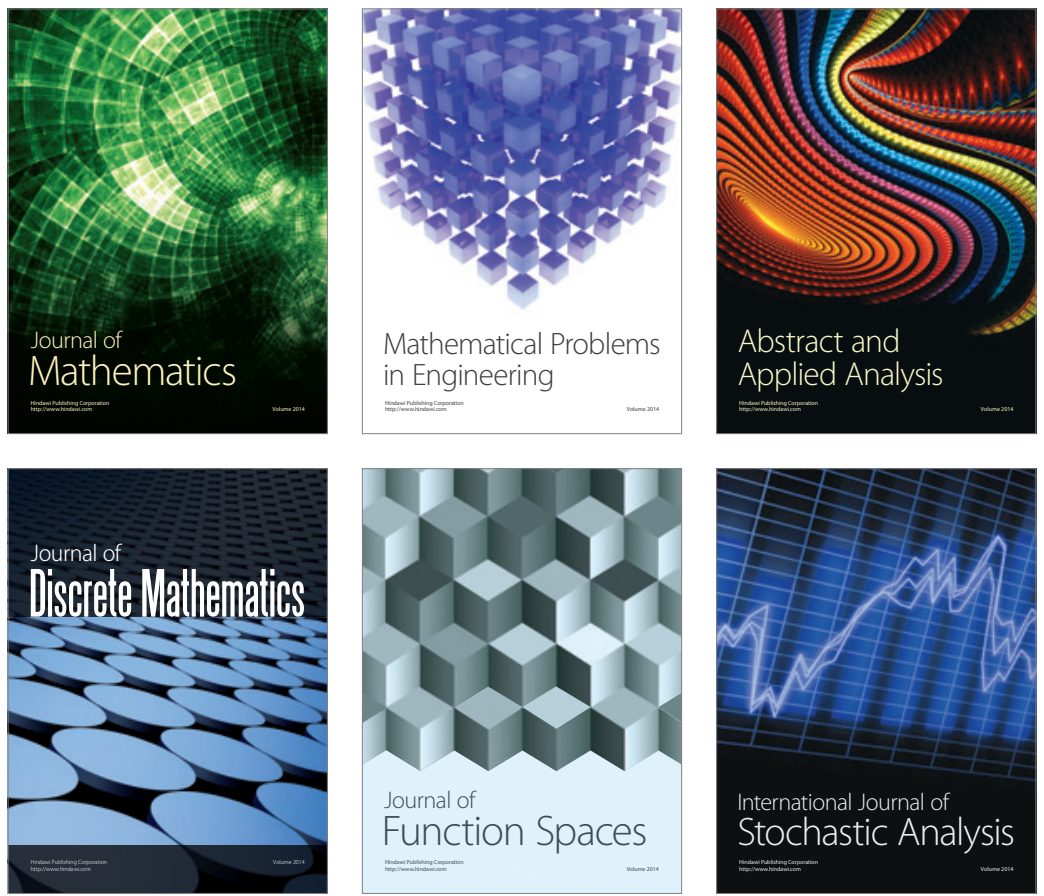

Journal of

Function Spaces

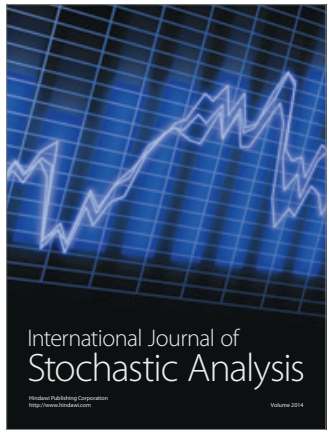

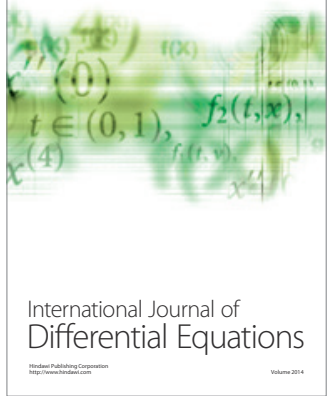
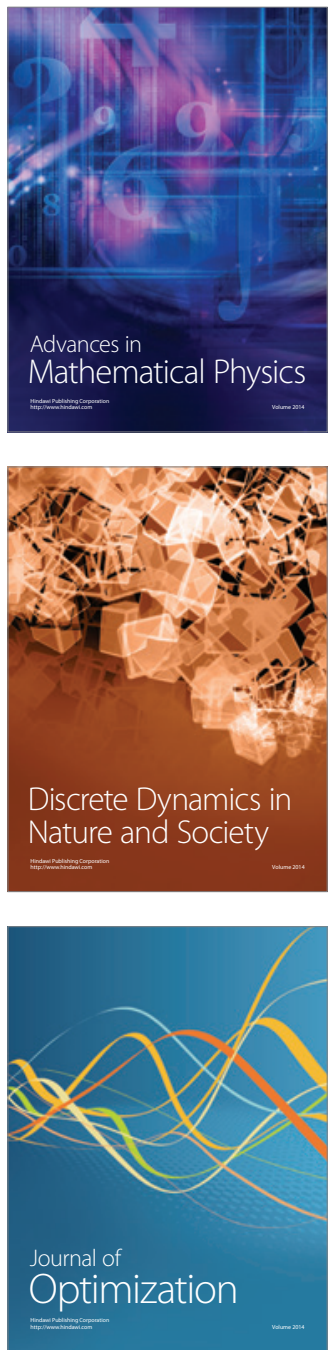